\title{
Analysis of the Behavior of a HV AC Line during an Atmospheric Discharge and Evaluation of the Spatio-Temporal Distribution of the Lightning Current Wave
}

\author{
Anthony Bassesuka Sandoka Nzao \\ ISTA Kinshasa, Electrical Engineering, Kinshasa, Democratic Republic of the Congo \\ Email: bass_sandoka@yahoo.fr
}

How to cite this paper: Nzao, A.B.S. (2022) Analysis of the Behavior of a HV AC Line during an Atmospheric Discharge and Evaluation of the Spatio-Temporal Distribution of the Lightning Current Wave. Open Journal of Applied Sciences, 12, 175-195. https://doi.org/10.4236/ojapps.2022.122013

Received: November 26, 2021

Accepted: February 15, 2022

Published: February 18, 2022

Copyright $\odot 2022$ by author(s) and Scientific Research Publishing Inc. This work is licensed under the Creative Commons Attribution International License (CC BY 4.0).

http://creativecommons.org/licenses/by/4.0/

\begin{abstract}
In high voltage networks for the transport of electrical energy, lightning, a phenomenon as dangerous as it is impressive, with an easily recognizable form, can affect a power line by striking either a phase conductor, a tower or a guard cable, thus causing more dangerous and constraining stresses on the lines for its proper operation. Thus, this article aims to analyze the behavior of a HV line during an atmospheric discharge and assess the spatial and temporal distribution of the lightning current wave. For this purpose, the generalities on the transmissible power in case of link without resistance and the modeling of the atmospheric surge propagation established on the basis of the theory of the lines with distributed constants implementing the wave equation known as the Alembert equation have been developed. Through this research, we are interested in the study of the space-time distribution of the lightning current wave in order to model the radiated electromagnetic field and to examine the influence of the atmospheric discharge induced overvoltage on the transportable power of a High Voltage AC Transmission line, for a good selective protection in order to illuminate the parasites. The $2 \mathrm{D}$ simulation based on engineering and "Transmission Line" models have been developed as well as the verification of the coherence of the different models, by comparing the fractal dimensions of the program results with those of the experimentally obtained figures.
\end{abstract}

\section{Keywords}

Analysis, Lightning, HV Line, Pylons, Kirchhoff Equations, Alembert Equation, Engineering Model, Transmission Line 


\section{Introduction}

The considerable development of the electric power transmission network in its various components which ensure an optimal distribution of power transits, the knowledge in real time of the characteristic electric quantities of the latter makes it possible to ensure its control and command and leads progressively to the study of the various disturbances [1]; by their mode of transmission such as the phenomenon of wave propagation which is the basis of many cases of information transmission such as Hertzian waves, telecommunication networks, energy distribution networks, lightning, nuclear electromagnetic pulse [2]; by their form in particular, interruptions, flicker phenomenon and high frequencies [3]; and by the fact that they affect electrical organs or human [4].

By order of priority and rate of impact in the classes of constraint, lightning represents the unpredictable natural phenomenon and the most harmful on all electro-energetic systems [5] and if we can affirm that nowadays, the energy transporters control adequately the protection of the network against the internal accidental defects, it is not the case for its protection against lightning, especially during an indirect impact where it radiates important electromagnetic fields and which will induce by electromagnetic coupling cruel surges in their targets, in particular the electric power transmission networks [6]. External over voltages propagating in the electrical network is likely to cause dielectric breakdowns in the materials which ensure the insulation of the network components [7], the drop in active transmissible power being able to cause the unscheduled cut for the case of the lines functioning without the margin of reserve in power etc. [8]. And these, therefore, require a detailed study of the propagation of these over voltages, assumed to be the most severe stresses on electro-energetic systems, so as to predict the level that they can reach at any point of the electrical network in order to recommend adequate solutions [9]. The main concern today for both operators and consumers is to control the transmission parameters, especially in $\mathrm{AC}$, to ensure stability, to increase the efficiency of the transmission system and to improve the efficiency of the transmission system, especially in AC. The major concern today for both operators and consumers and the transmission of energy whose main element is the power line is to control the parameters of the transmission, especially in alternating current, to ensure stability, increase efficiency and illuminate the parasites for a good selective protection [10]. Given the complexity of the study of the parameters of a line and given that the number of its parameters introduced several unknowns; we are limited to the study of the fluctuations of the transportable power for a compensated inductive line in order to approach the reality.

\section{Study Methodology}

Through this research, we are interested in the Analysis of the Behavior of an AC HV Line during an. Atmospheric Discharge and in the Evaluation of the Spatio-Temporal Distribution of the Lightning Current Wave. Transmission 
lines are the most exposed to disturbances during a lightning discharge. Their transient behavior study is generally based on the theory of transmission lines with R, L, C and G [11] electrical components. Several equations exist for their modeling depending on the shape and the characteristic of the material used in the installation. In this paper, the bi-exponential models [12] and the so-called engineering models [13] [14] have been chosen and used for the analytical modeling of the spatio-temporal lightning current distribution. The 2D simulations based on the proposed models have been presented as well as the verification of the coherence of the different modelling, by comparing the fractal dimensions of the results of our programs with those of the figures obtained experimentally. To this end, the organization of this paper follows the following process:

\section{Study and Modeling of Power Transit on HV Lines}

\subsection{Transmittable Power in the Case of a Connection without a Resistor}

The voltage of a line must remain fairly constant as the active power consumed by the customers varies. Generally, the voltage variation from zero to full load should not exceed a certain value ranging from $5 \%$ to $10 \%$ of the nominal voltage depending on the type of system. The maximum power that a line can carry also depends on the voltage variation [10]. Here, the study is made on a compensated inductive line. Considering an inductive line with negligible resistance but which has an inductive reactance $X$ and a capacitive reactance $X_{c}$. The voltage $V$ measured at the end of the line decreases as the load increases [15].

$$
V=E-X I
$$

With: $E$ : the electromotive force in Volt.

The relationship (1) between the voltage $V$ at the load busbar and the load current $I$ is called the load line of the system which defines the equation of a straight line passing through $V$ and having the slope as shown in the following Figure 1.

As a reminder, this model only makes sense in the case of a steady state. We also consider that this machine is connected to a so-called "infinite" network, i.e. whose voltages and phase remain constant whatever the disturbances whatever the disturbances imposed [16]. The operation of this system is then described by Equation (2), written in polar notation. The network represents, here, the synchronous reference. This results in the vector diagram of Figure 2 below.

$$
E-j X . I=V_{m}
$$

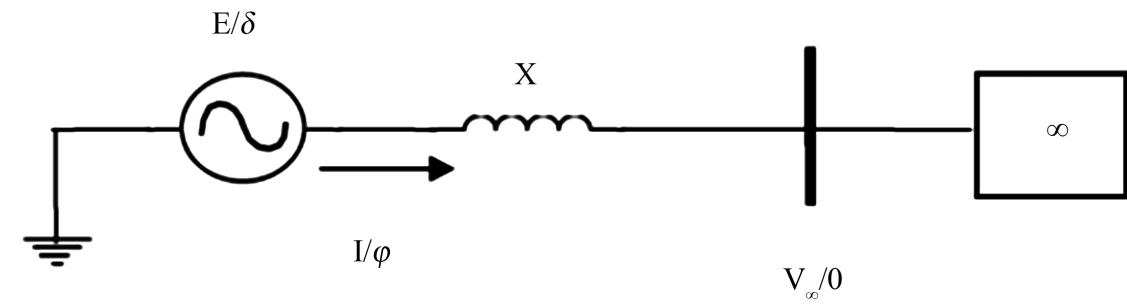

Figure 1. Diagram of an inductive line. 


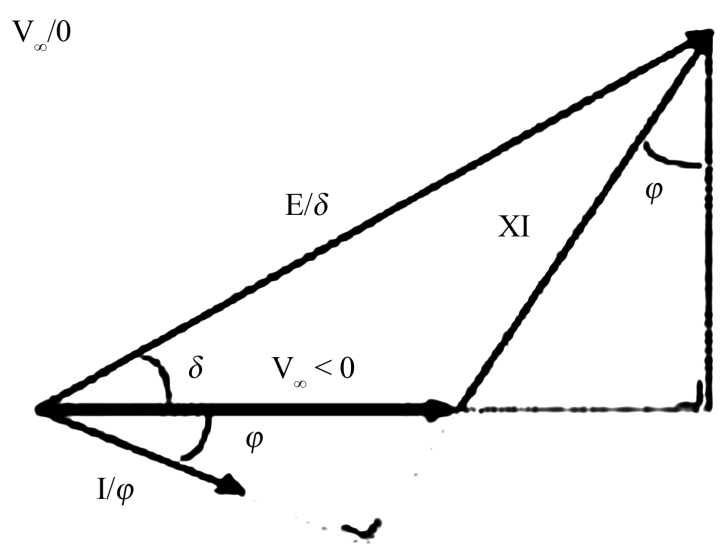

Figure 2. Vector diagram of electrical quantities.

We can improve the regulation and increase the transportable power by adding a capacitive reactance $X_{c}$ at the end of the line. The voltage $V$ can be kept constant by adding the value of $X_{c}$ so that the reactive power supplied by the capacitor see expression (3) below is equal to half the reactive power absorbed by the line see expression (4) below [17]:

$$
\begin{gathered}
P_{f}=\frac{U^{2}}{X_{c}} \\
P_{a b}=X_{c} I^{2}
\end{gathered}
$$

With: $P_{f}$ : power supplied by the capacitor in $\mathrm{kVAr}$ and $P_{a b}$ : reactive power absorbed by the line in $\mathrm{kVAr}$.

However, we see that there is still an upper limit $P_{\max }$ to the active power that the line carries.

$$
P_{a c}=\frac{E}{x}\left(2 E-V_{\infty}\right) \cdot \sin \delta
$$

With: $P_{a c}$ Upper limit to the active power that the line carries in MW.

This relationship (5) shows the transmittable power does a function of the phase shift angle " $\delta$ " exist between the machine and the infinite network. The graph of this power as a function of the phase shift, is then half sinusoid see Figure 3 below [18].

Points " $S$ " such that " $\delta$ " is less than $\pi / 2$ are stable, while points " $P$ " for which " $\delta$ " $\in[\pi / 2, \pi]$ are unstable. This can be explained by the following two remarks: Let " $P_{m}$ " be the mechanical power supplied to the generator, cfr Figure 3 [19]:

- For " $\delta<\pi / 2$ ", if a small disturbance occurs, tending to increase respectively decrease the rotor angle, the electric power produced becomes greater or less than the mechanical power rotor angle, the electric power produced becomes higher or lower than the mechanical power. Given the equation of motion of the synchronous machine, the angle $\delta$ tends to decrease as " $\frac{\mathrm{d}^{2} \delta}{\mathrm{d} t^{2}}<0$ ".

In both cases, there is a propensity for the system to return to equilibrium. 


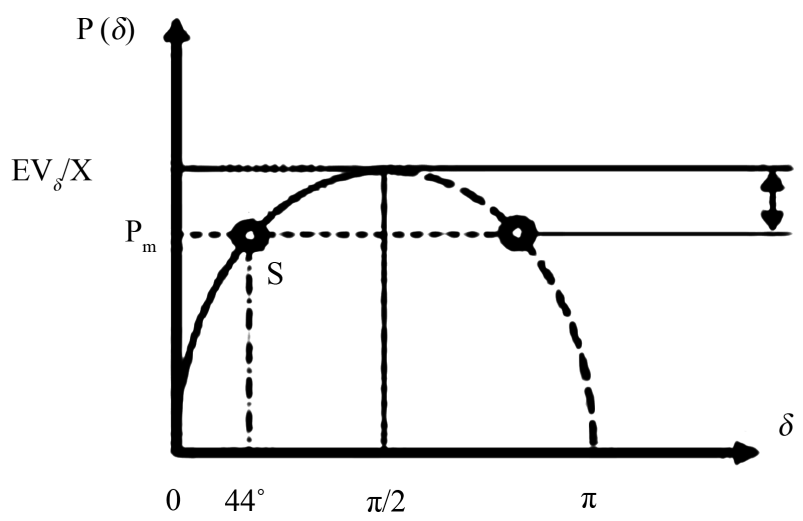

Figure 3. Transmissible power as a function of the phase shift between current and voltage.

- For " $\delta>\pi / 2$ ", the behavior is opposite: moved away from its equilibrium position, the rotor tends to move away from it further away! Although, in theory, the stability zone extends from 0 to $\pi / 2$, in practice we are limited to a value of the order of $\delta_{\max } \sim 30^{\circ}$ to $35^{\circ}$, for a heavily loaded network. The stability margin thus conserved allows us to maintain the system in a stable state, even in case of a transient disturbance such as a change in a modification of the load distribution during a network reconfiguration, a modification of the energy generation, or even in case of a temporary fault or loss of the generating set.

Note: A distinction must be made between the angle " $\delta$ " between the voltages of two neighboring network nodes and the angle between two. The value of the latter can be as high as $45^{\circ}$ [20].

\subsection{Transmissible Power (Resistance-Free Connection)}

In the equivalent diagram of Figure 4 below, we neglect, as a first approximation, the effect of the capacitances capacities and the lines own resistance. The latter is then only represented by an inductance $X$ as shown in the following diagram [21]:

By reasoning similar to the previous one, we deduce that the power transfer is guided by expression 4 . These results can also be obtained from the load distribution equations.

$$
P=\frac{u_{s}^{2}}{x} \cdot \sin \delta
$$

Let be:

$$
P=\frac{u_{s}}{X}\left(2 u_{s}-u_{R}\right) \cdot \sin \delta
$$

With: $P$. transmissible power through the inductive line in MW; $u_{s}$ : voltage of the source in $\mathrm{kV}$ and $u_{R}$ : voltage at the line output or load terminals $\mathrm{kV}$.

Disturbances in the network load pattern are directly reflected in this angle $\delta$, which forces one to keep it, for stability reasons, below a value generally below 


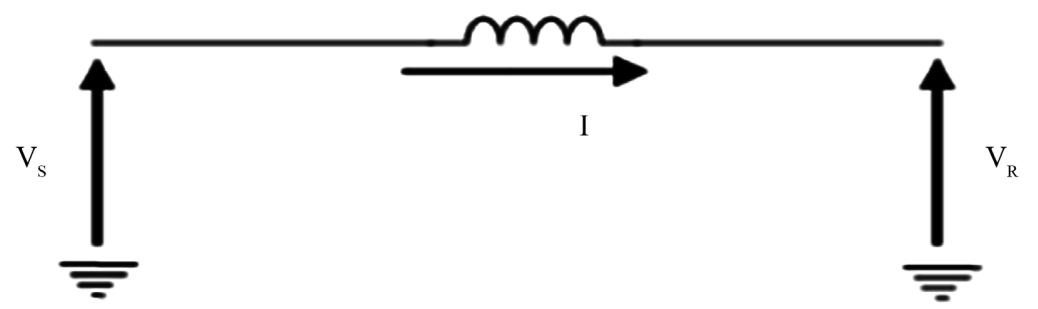

Figure 4. Simplified diagram of the line.

$30^{\circ}-35^{\circ}$. For a link loaded at its natural power, this angle puts a limit to the series reactance, and thus sets a limit length.

The maximum power $P_{\max }$ is reached if $\delta=30^{\circ}$ and $u_{R}=u_{s}$.

$$
P=\frac{u_{s}^{2}}{2 X}
$$

Caption:

$P$ : Maximum power in MW;

$u_{s}$ : Source voltage in $\mathrm{kV}$;

$X$ : Reactance of the line in Ohm.

\subsection{Study of Air Discharge on an Airline}

Transmission lines are the most exposed to disturbances during a lightning discharge. Their transient behavior is generally based on the theory of transmission lines with $R, L, C$ and $G$ [11] electrical components. Several equations exist for their modeling depending on the shape and the characteristic of the material used in the installation. In this paper, the bi-exponential [12] and the so-called engineer's models [13] [14] have been chosen and used for the analytical modeling of the spatio-temporal lightning current distribution.

The calculation of the electromagnetic field requires in this case:

- A model of the lightning current measured at the base of the channel.

- A spatio-temporal modeling of the current distribution in the lightning channel.

\subsubsection{Lightning Current Analytical Modeling}

To model lightning, it is considered as a current wave varying in time. The oldest and simplest analytical model is a difference of two decreasing exponentials. This model has the advantage of having analytical Fourier transforms, which allows a direct analysis in the frequency domain [12]. It was proposed by Bruce and Golde in 1941 [Cooray V., 2003] [22] and is represented by the following formula:

$$
i(t)=I_{o}\left(\mathrm{e}^{-A t}-\mathrm{e}^{-B t}\right)
$$

$I_{o}$ : Is the amplitude of the current in the lightning channel, $A$ and $B$ are rise and fall time constants respectively.

The Heidler function is also the analytical expression for the current at the base of the lightning channel presented in 1985 [12]. The use of the latter has yielded results that are more consistent with experimental observations. Mathematically, this function is modelled as follows: 


$$
i(0, t)=\frac{I_{o}}{\eta} \frac{\left(\frac{t}{\tau_{1}}\right)^{n}}{1+\left(\frac{t}{\tau_{1}}\right)^{n}} \mathrm{e}^{\left(\frac{-t}{\tau_{2}}\right)}
$$

Caption:

$I_{o}$ : Amplitude of the base channel current;

$\tau_{1}:$ Rise time constant;

$\tau_{2}$ : Downward time constant;

$\eta$ : Wave amplitude correction factor and;

$n$ : is an exponent varying between 2 and 10 .

Below are the lightning current parameters from relationship (10), for $n=10$.

$$
i(0, t)=\frac{I_{01}}{\eta_{1}} * \frac{\left(\frac{t}{\tau_{11}}\right)^{n_{1}}}{1+\left(\frac{t}{\tau_{21}}\right)^{n_{1}}} \mathrm{e}^{\left(\frac{-t}{\tau_{22}}\right)}+\frac{I_{02}}{\eta_{2}} * \frac{\left(\frac{t}{\tau_{11}}\right)^{n_{2}}}{1+\left(\frac{t}{\tau_{21}}\right)^{n_{2}}} \mathrm{e}^{\left(\frac{-t}{\tau_{22}}\right)}
$$

\subsubsection{Modeling the Current Distribution in the Lightning Channel}

The calculation of the electromagnetic field generated by lightning requires the knowledge of the lightning current along the channel 26. For this purpose, several models have been developed due to the complexity of the current propagation phenomenon in the ionized channel in order to reproduce the physical phenomena as well as possible. However, these models can be divided into two categories:

- Engineering models;

- Antenna models.

In this case, we will limit ourselves to the Bruce and Golde (BG) models proposed in 1941, the "Transmission Line" (TL) models in 1969 and the antenna type models.

\section{1) Bruce and Golde (BG) model (1941)}

This is one of the first models of its kind and probably the simplest. According to this model, the current $i\left(z^{\prime}, t\right)$ at heights below the return arc front is equal to the current at the base of the lightning channel, and at heights above the return arc front is zero [23] [24]:

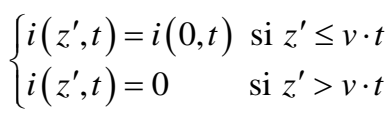

where: $v$ is the propagation speed of the lightning wave in $\mathrm{m} / \mathrm{Sec}$.

\section{2) Transmission Line (TL) model}

This model was presented by Uman and Mclain [25] [26] [27]. This model assimilates lightning to a transmission line. It assumes that the arc back current propagates along the lightning channel. The current distribution is defined by:

$$
\left\{\begin{array}{l}
i\left(z^{\prime}, t\right)=i\left(0, t-\frac{z^{\prime}}{v}\right) \quad \text { si } z^{\prime} \leq v \cdot t \\
i\left(z^{\prime}, t\right)=0 \text { ou } i\left(z^{\prime}, t\right)<0 \text { si } z^{\prime}>v \cdot t
\end{array}\right.
$$


Combining Equations (9) and (13), we obtain:

$$
i\left(t-\frac{z^{\prime}}{v}\right)=I_{o}\left[\mathrm{e}^{-A\left(t-\frac{z^{\prime}}{v}\right)}-\mathrm{e}^{-B\left(t-\frac{z^{\prime}}{v}\right)}\right]
$$

Equation (14) defines the space-time distribution of lightning current. This lightning current distribution depends on time $t$ and space $z$.

\section{3) The antenna type model}

Frequency-domain antenna theory has been used very recently to study the coupling between the field radiated by lightning and electrical networks. The solution of the integral equation is usually done by the method of moments which consists in associating the integral equation with a system of linear algebraic equations equivalent to a matrix equation. In the formalism, the lightning channel is represented by thin wires to determine the current distribution. However, an important constraint of the antenna theory is the computational time which can become prohibitive very quickly [14].

\subsubsection{Calculation of the Electromagnetic Fields Radiated by a Storm Discharge}

The calculation of the electromagnetic field requires:

- A model of the lightning current measured at the base of the channel.

- A spatio-temporal modeling of the current distribution in the lightning channel.

The calculation of the electromagnetic field will be carried out by adopting several simplifying hypotheses, and by using the formalism of the Hertzian dipoles which consist in subdividing the channel into a succession of elements called [27]. The total field emitted by the channel is obtained by superimposing all the dipoles. In reality, the lightning channel is not rectilinear, and comprises a succession of small segments whose directions would follow a Gaussian distribution [26] [27] [28] [29]. Given the randomness of this tortuosity, we will limit ourselves here to the simple case of the one-dimensional vertical channel of height $H$, as illustrated in Figure 5. The return arc propagates from the ground with a velocity $V$, the space-time distribution of the current through the channel determines the electromagnetic field at any point in space.

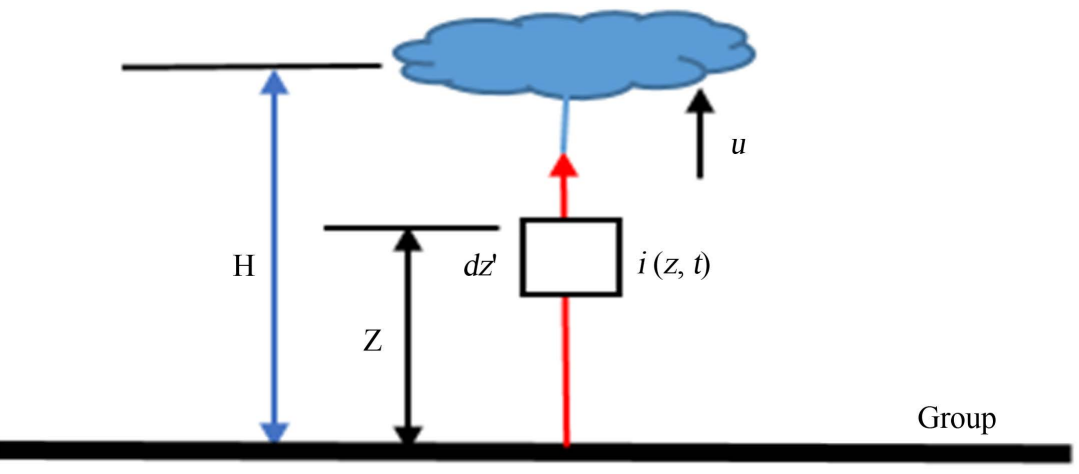

Figure 5. Lightning channel representation. 
The calculation of the EM field radiated by a dipole placed above the ground of finite conductivity was first presented by Sommerfeld in 1909 [30]. These equations written in the frequency domain contain so-called Sommerfeld integrals. Furthermore, the need to perform an inverse Fourier transform can cause numerical problems. Thus, in order to calculate the electromagnetic field radiated by the lightning channel, we will use as a starting point the method proposed by Rachidi in 1991 [31]. This method allows us to perform the calculations quickly with good accuracy. It is performed in two steps:

- At first, we assume the earth as a perfect conductor.

- After this first approximation, the soil conductivity is taken into account by correcting the values obtained for a perfect soil.

\subsubsection{Above Ground Field Model}

\section{Case of a perfectly conductive soil}

We assume that the lightning current propagates towards the clouds along the " $z$ " axis. The total field radiated at a point in space is thus obtained by summing the contributions of each dipole and its image of length $d z$, located at height ${ }^{\star} Z^{\star}$, as shown in Figure 6, the size of the dipoles must satisfy two conditions [29] [31]:

$d z \leq \frac{\lambda}{20}, \lambda:$ is the pseudo wavelength of the transient phenomenon and $\leq \frac{R}{20}, R$ : The radius of observation.

Approximating the lightning channel to a straight wire antenna perpendicular to a perfectly conducting infinite plane, we can then express the radiation of each dipole and its image at a point P located at a distance $r$ and an altitude $z$ by the Formulas (15), (16) and (17)

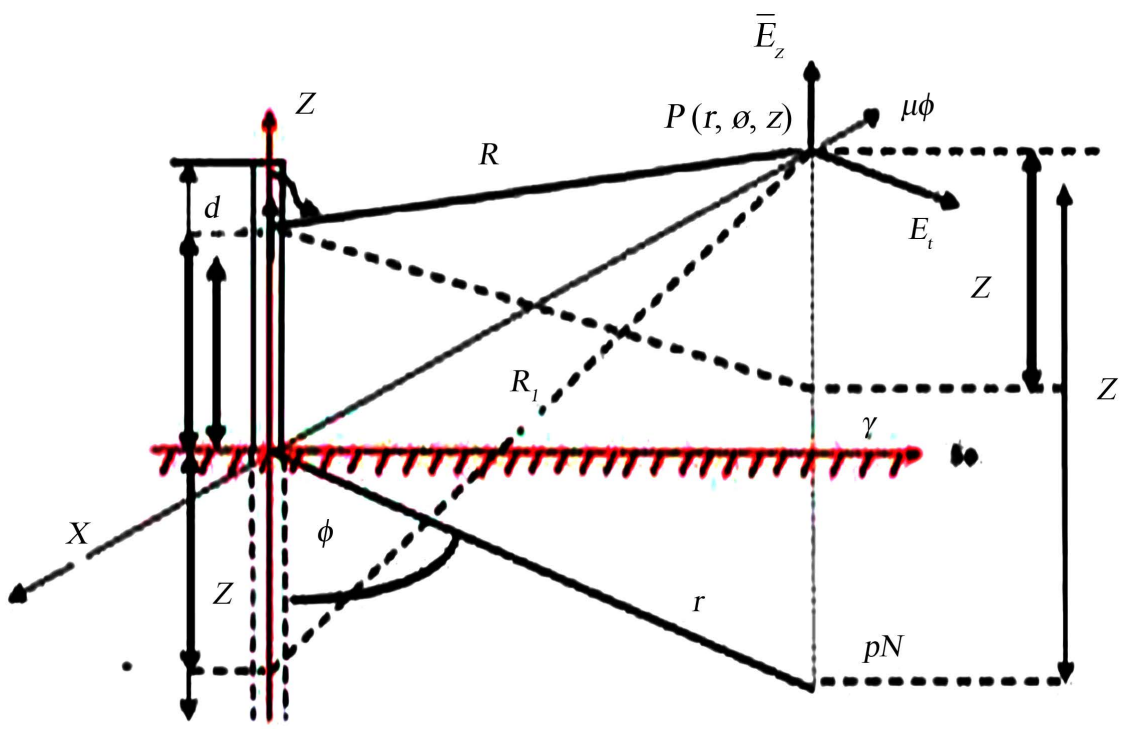

Figure 6. The model for calculating the EM field radiated over a perfectly conducting ground. 


$$
\begin{gathered}
\mathrm{d} E_{z}(r, z, t)=\frac{\mathrm{d} z^{\prime}}{4 \pi \varepsilon_{0}}\left[\frac{2\left(z-z^{\prime}\right)^{2}-r^{2}}{R^{5}} \int_{0}^{t} i\left(z^{\prime}, \tau-\frac{R}{c}\right) \mathrm{d} \tau\right. \\
\left.+\frac{2\left(z-z^{\prime}\right)^{2}-r^{5}}{c R^{4}} i\left(z^{\prime}, \tau-\frac{R}{c}\right)-\frac{r^{2}}{c^{2} R^{3}} \frac{\partial}{\partial t} i\left(z^{\prime}, \tau-\frac{R}{c}\right)\right] \\
\mathrm{d} E_{r}(r, z, t)=\frac{\mathrm{d} z^{\prime}}{4 \pi \varepsilon_{0}}\left[\frac{3 r\left(z-z^{\prime}\right)^{2}-r^{2}}{R^{5}} \int_{0}^{t} i\left(z^{\prime}, \tau-\frac{R}{c}\right) \mathrm{d} \tau\right. \\
\left.+\frac{3 r\left(z-z^{\prime}\right)^{2}-r^{2}}{c R^{4}} i\left(z^{\prime}, \tau-\frac{R}{c}\right)-\frac{r\left(z-z^{\prime}\right)^{2}}{c^{2} R^{3}} \frac{\partial}{\partial t} i\left(z^{\prime}, \tau-\frac{R}{c}\right)\right] \\
\left.\mathrm{d} B_{\varphi}(r, z, t)=\frac{\mu_{0} \mathrm{~d} z^{\prime}}{4 \pi}\left[\frac{r}{R^{3}} i\left(z^{\prime}, \tau-\frac{R}{c}\right)-\frac{r}{c R^{2}} \frac{\partial}{\partial t} i\left(z^{\prime}, \tau-\frac{R}{c}\right)\right]\right] \\
R=\sqrt{r^{2}+\left(z-z^{\prime}\right)^{2}}
\end{gathered}
$$

$r$. The distance between the impact point and the observation point.

$z$ : The height of the observation point.

$(z, t):$ The current along the channel.

$c$ is the speed of light.

$\mu_{0}, \varepsilon_{0}$ : are the permeability and permittivity of vacuum, respectively.

The three terms involved in Equations (15) and (16), represent the electrostatic (they are the dominant components of the field near the source), induction and radiation fields (they are the dominant components of the field far from the source) respectively. While the first term in Equation (17) represents the induction field "or magneto static field" (the dominant one near the source), and the second is the radiation field. The total fields produced by the return arc current are obtained by the integral of the equations along the channel, or the spatial and temporal distribution of the current.

\subsubsection{Field-in-Ground Model}

The determination of the disturbances induced by a lightning strike in buried cables requires the knowledge of the electromagnetic field radiated by the lightning in the ground. For this purpose, we will adapt the geometry of Figure 7 to this problem.

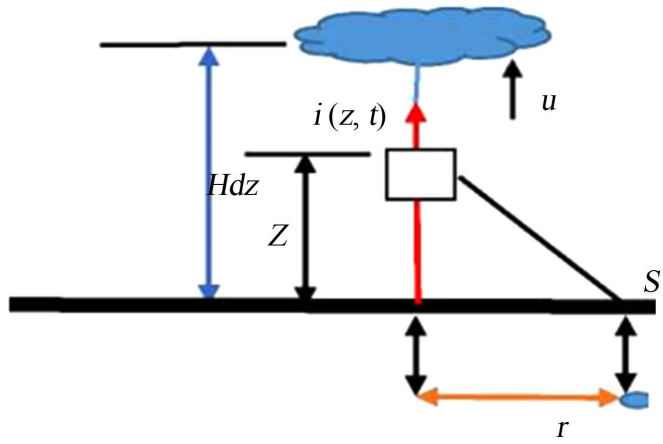

Figure 7. The model for calculating the EM field radiated into the ground. 
Considering the lightning channel as a vertical antenna above a soil of finite conductivity, the general expressions for the field created by an elementary dipole of the lightning channel in frequency domain where $p=j \omega$ located at a height " $z$ " above the ground, distance " $r$ " and depth " $d$ " are given by:

$$
\begin{gathered}
\mathrm{d} E_{r}\left(r, d, z^{\prime}\right)=\frac{j \omega \mu_{0} I\left(z^{\prime}\right) \mathrm{d} z^{\prime}}{4 \pi} \frac{\partial^{2} V\left(r, d, z^{\prime}\right)}{\partial r \partial z} \\
\mathrm{~d} E_{z}\left(r, d, z^{\prime}\right)=\frac{j \omega \mu_{0} I\left(z^{\prime}\right) \mathrm{d} z^{\prime}}{4 \pi}\left(\frac{\partial^{2} V\left(r, d, z^{\prime}\right)}{\partial z^{2}}+k_{g}^{2}\right) V\left(r, d, z^{\prime}\right) \\
\mathrm{d} H_{\varphi}\left(r, d, z^{\prime}\right)=\frac{-I\left(z^{\prime}\right) \mathrm{d} z^{\prime}}{4 \pi}\left(\frac{\partial k_{g}^{2} V\left(r, d, z^{\prime}\right)}{\partial r}\right) \\
V\left(r, d, z^{\prime}\right)=\int_{0}^{\infty} \frac{\mathrm{e}^{\gamma_{g} d-\gamma_{0} z^{\prime}}}{k_{g}^{2} \gamma_{0}+k_{g}^{2} \gamma_{g}} j_{0}\left(\lambda_{r}\right) \lambda \mathrm{d} \lambda
\end{gathered}
$$

With: $\omega=2 \pi f$ and $k_{0}=\omega^{2} \mu_{0} \varepsilon_{0}$,

For $p=j \omega$ La place variable:

$I\left(z^{\prime}\right)$ denotes the Fourier transform of the current distribution $i\left(z^{\prime}, t\right)$ along the channel, and $k_{g}$ and $k_{0}$ the propagation constants in the ground and air respectively. The components of the total EM field are obtained by integrating expressions (19), (20) and (21) along the channel. In addition, if we are interested in the temporal evolution of the field, it is necessary to perform an inverse Fourier transform.

The analysis of the coupling of a lightning wave with a wire structure leads us to express the electric and magnetic fields in a Cartesian frame by their three components $\left(E_{x}, E_{y}\right.$ and $\left.E_{z}\right)$ and $\left(H_{x}, H_{y}\right.$ and $\left.H_{z}\right)$ given by the following transformations:

$$
\begin{aligned}
& \left(\begin{array}{l}
\mathrm{d} E_{x} \\
\mathrm{~d} E_{y} \\
\mathrm{~d} E_{z}
\end{array}\right)=\left(\begin{array}{cc}
\cos \varphi & 0 \\
\sin \varphi & 0 \\
0 & 1
\end{array}\right)\left(\begin{array}{l}
\mathrm{d} E_{r} \\
\mathrm{~d} E_{z}
\end{array}\right) \\
& \left(\begin{array}{l}
\mathrm{d} H_{x} \\
\mathrm{~d} H_{y} \\
\mathrm{~d} H_{z}
\end{array}\right)=\left(\begin{array}{c}
-\sin \varphi \\
\cos \varphi \\
0
\end{array}\right)\left(\mathrm{d} H_{\varphi}\right)
\end{aligned}
$$

The essential requirements of Electromagnetic Compatibility (EMC) aim to ensure optimal operation of electronic systems with respect to external electromagnetic phenomena. Lightning is the main natural phenomenon that generates electromagnetic disturbances. Power lines are subject to disturbances of atmospheric origin (lightning). These disturbances lead to the propagation of atmospheric overvoltage, which can modify the behavior of HV lines and cause tripping that is detrimental to service continuity. For this purpose, in the following lines, we have developed the atmospheric surge propagation model on the HV AC power transmission line. 


\subsection{Atmospheric Surge Propagation Modeling}

\section{Analytical Model}

Surges are high frequency moving waves, so the modeling of their propagation must use a distributed constant line description [32]. This means that the resistive, inductive and capacitive components of the line have characteristics per unit length and can be symbolized by: $R^{\prime}, L^{\prime}, C$ and $G$. In our model, the line is assumed to be bifilar, each element of elementary length $\mathrm{d} x$, the line is characterized by an elementary resistance $R^{\prime} \mathrm{d} x$ in series with an elementary choke $L^{\prime} \mathrm{d} x$, and in parallel, by an elementary conductance $G \mathrm{~d} x$ in turn in parallel with an elementary capacitance $C \mathrm{~d} x$, see Figure 8 [33].

Applying the Kirchhoff and network node rule to the lossy line element in Figure 8, we write:

$$
\begin{aligned}
& i(x+\Delta x, t)=i(x, t)-G^{\prime} \mathrm{d} x \cdot u(x, t)-C_{d}^{\prime} \mathrm{d} x \cdot \frac{\delta u(x, t)}{\delta t} \\
& u(x+\Delta x, t)=u(x, t)-R^{\prime} \mathrm{d} x \cdot i(x, t)-L_{d}^{\prime} \mathrm{d} x \cdot \frac{\delta i(x, t)}{\delta t}
\end{aligned}
$$

This gives after expansion of $u(x+\mathrm{d} x, t)-u(x, t)$ and $i(x+\mathrm{d} x, t)-i(x, t)$, the differential equations of the voltage and current, called the Alembert's equations as follows:

$$
\begin{gathered}
\frac{\delta i(x, t)}{\delta x}=-G^{\prime} \cdot u(x, t)-C_{d}^{\prime} \cdot \frac{\delta u(x, t)}{\delta t} \\
\frac{\delta u(x, t)}{\delta x}=-R^{\prime} \cdot i(x, t)-L_{d}^{\prime} \cdot \frac{\delta i(x, t)}{\delta t}
\end{gathered}
$$

which can be put together in either expression of the so-called telegraphers' equation:

$$
\begin{gathered}
\frac{\delta^{2} u(x, t)}{\delta x^{2}}-L_{d}^{\prime} \cdot C_{d}^{\prime} \cdot \frac{\delta^{2} u(x, t)}{\delta t^{2}}-\left(R^{\prime} \cdot C_{d}^{\prime}-L_{d}^{\prime} \cdot G^{\prime}\right) \frac{\delta u(x, t)}{\delta t}-R^{\prime} \cdot G^{\prime} \cdot u(x, t)=0 \\
\frac{\delta^{2} i(x, t)}{\delta x^{2}}-L_{d}^{\prime} \cdot C_{d}^{\prime} \cdot \frac{\delta^{2} i(x, t)}{\delta t^{2}}-\left(R^{\prime} \cdot C_{d}^{\prime}+L_{d}^{\prime} \cdot G^{\prime}\right) \frac{\delta i(x, t)}{\delta t}-R^{\prime} \cdot G^{\prime} \cdot i(x, t)=0
\end{gathered}
$$

The Equations (29) and (30) represent the atmospheric surge propagation model on a transmission line power line, containing the components such as: $R$ : (Resistance in Ohm per kilometer), $L$ : (Inductance in Henry per kilometer), $C$ : (Capacitance in Faraday per kilometer) and $G$ : (Conductance in Siemens per kilometer). For the case of a lossless line, i.e., $R=G=0$, expressions (29) and (30) can be written as follows:

$$
\begin{aligned}
& \frac{\delta^{2} u(x, t)}{\delta x^{2}}-L_{d}^{\prime} \cdot C_{d}^{\prime} \cdot \frac{\delta^{2} u(x, t)}{\delta t^{2}}=0 \\
& \frac{\delta^{2} i(x, t)}{\delta x^{2}}-L_{d}^{\prime} \cdot C_{d}^{\prime} \cdot \frac{\delta^{2} i(x, t)}{\delta t^{2}}=0
\end{aligned}
$$

The analytical solution of expression (31) as a function of $\mathrm{x}$ and time $\mathrm{t}$ assuming that the wave is quasi-stationary is given by the following mathematical model: 


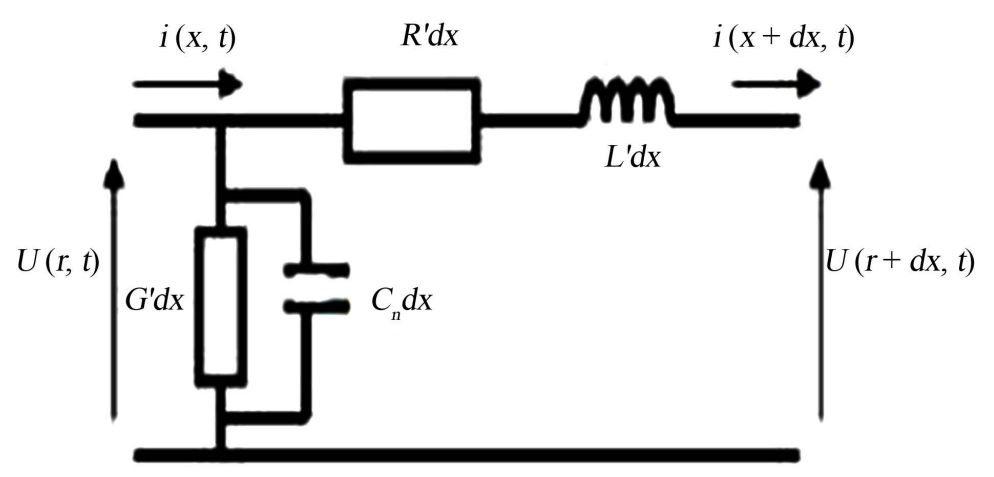

Figure 8. Model of an elementary length $d x$, of a line with distributed constants.

$$
u(x, t)=u_{s} \cdot \exp (-\alpha x) \cdot \sin [\omega t+\phi] g(x)
$$

Here we assume that the frequency does not vary, the product $L^{\prime} \cdot C_{d}^{\prime}=\omega^{2}=L^{\prime} \cdot C_{d}^{\prime}=\alpha$, is called the propagation time constant of the surge voltage on the transmission line. In the expression (33), we see transient component $g(x)$ which can bring the voltage to a very large value during a half period of overvoltage.

\section{Analysis of Lightning Strike on an Overhead Power Line}

Several cases can occur, in particular the lightning strike on the phase conductor, the lightning strike on the guard wire (in full span or at the top of the tower) and the lightning strike on the ground near an overhead line. The last case does not interest us for lines with a voltage higher than $90 \mathrm{kV}$ because the over voltages rarely exceed $600 \mathrm{kV}$, a value lower than the insulation level of these lines [Kiessling F., Nefzger P., Nolasco J.F., Kaintzyk U., 2003 [34]. In the present case, we will focus on the first case, i.e. the lightning strike directly on the phase conductor, this aspect will allow us to visualize the impact of the overvoltage due to the atmospheric discharge on the behavior of the power transportable by the HV AC line.

\section{Lightning Strike on a Phase Conductor}

When the lightning strike falls on the phase conductor of a line, the current $\mathrm{I}(\mathrm{t})$ in the conductor is distributed on both sides of the impact point along the conductor as modeled by Figure 9 below. And, it carries with it a surge wave whose value is quantifiable via the following relation:

$$
u_{C}=\frac{1}{2} \times Z_{C} \times i(t)
$$

With: $u_{C}$ Induced overvoltage in the phase conductor in $\mathrm{kV} ; Z_{C}$ : Wave impedance of the line in Ohm and $i(t)$ : Lightning current in A.

Combining Equations (9) and (34), we obtain the time model of the voltage wave induced by the lightning electromagnetic field in the phase conductor of an HV line: 


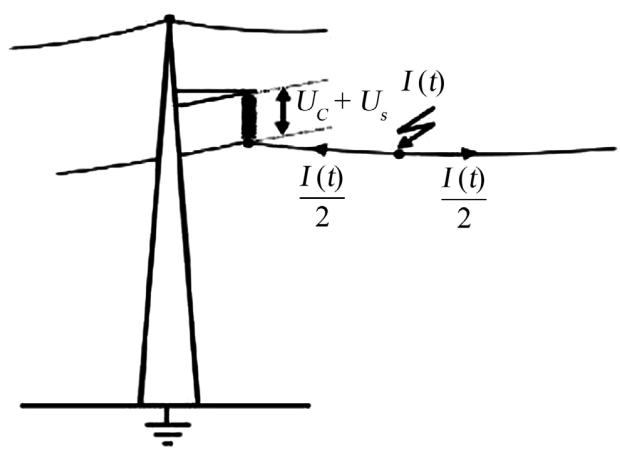

Figure 9. Lightning strike on a phase conductor of an overhead line.

$$
u_{C}=\frac{1}{2} \times Z_{C} \cdot I_{o}\left(\mathrm{e}^{-A t}-\mathrm{e}^{-B t}\right)
$$

At a given point on the line, for example at the first tower encountered by the wave, the voltage (Figure 10) increases until the insulator chain is eventually triggered (insulator bypass.

\section{Modeling the Power Behavior of a HV Line during Atmospheric Discharge}

\section{Power Model during Atmospheric Surge}

Starting from the analytical model of the power transmitted by the transmission line (see relation (7)) and considering the power transit angle at $30^{\circ}$ for stability reasons, for an atmospheric surge of the form (35) appearing at the line outlet, the power at the end of the line during this surge period can be modeled as follows:

$$
P=\left|\frac{u_{s}}{2 x}\left[2 u_{s}-u_{C}\right]\right|
$$

Expression (36) shows that, the increase in voltage at the output of the line ( $u_{C}$ : overvoltage $u_{R}$ : voltage at the output of the line) leads to a significant drop in the power supplied by this line.

\section{Numerical Simulation}

\subsection{Declaration of Model Parameters}

In this part of the paper we present in the tables below the numerical values of the model parameters outlined above. The values of parameters $A$ and $B$ of Equation (9) have been proposed in the work of Dennis and Pierce see Table 1 [25] [35], the parameters of the lightning current of relation (10), for $n=10$ are listed in Table 2 and Table 3 presents the standard parameters of Heidler functions corresponding to the first arc and the return arc of a typical lightning strike (Equation (11)).

The sequence of the different simulations takes into account the following conditions: 


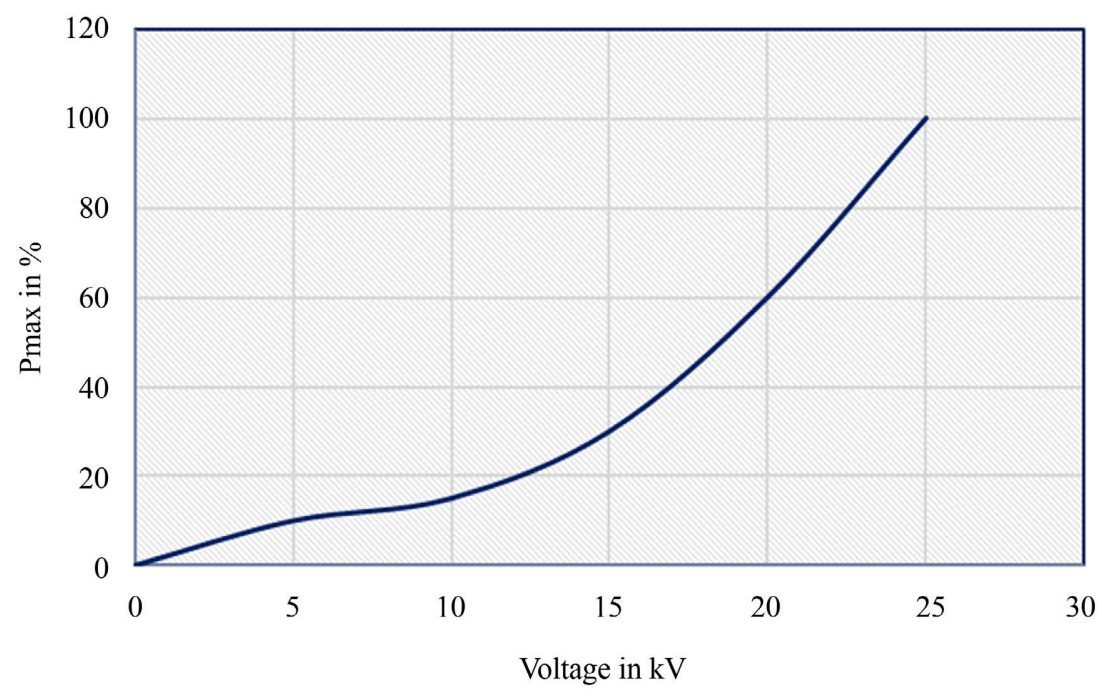

Figure 10. Power-voltage characteristic.

Table 1. Parameters of the bi-exponential lightning current model.

\begin{tabular}{cc}
\hline 1 st return arc & Subsequent return arc \\
\hline$I_{o}=30 \mathrm{kA}$ & $I_{o}=10 \mathrm{kA}$ \\
$A=2 \times 10^{4} \mathrm{~s}^{-1}$ & $A=1.4 \times 10^{4} \mathrm{~s}^{-1}$ \\
$B=2 \times 10^{5} \mathrm{~s}^{-1}$ & $B=6 \times 10^{5} \mathrm{~s}^{-1}$ \\
\hline
\end{tabular}

Table 2. Lightning current parameters.

\begin{tabular}{ccccccc}
\hline & \multicolumn{3}{c}{ First shot } & \multicolumn{3}{c}{ Consecutive short stroke } \\
\cline { 2 - 7 } Parameters & \multicolumn{3}{c}{ Level of protection } & \multicolumn{3}{c}{ Level of protection } \\
\cline { 2 - 7 } & I & II & III-IV & I & II & III-IV \\
\hline$I(\mathrm{kA})$ & 200 & 150 & 100 & 50 & 37.5 & 25 \\
$\eta$ & 0.93 & 0.93 & 0.93 & 0.993 & 0.993 & 0.993 \\
$\tau_{1}(\mu \mathrm{s})$ & 19 & 19 & 19 & 0.454 & 0.454 & 0.454 \\
$\tau_{2}(\mu \mathrm{s})$ & 485 & 485 & 485 & 143 & 143 & 143 \\
\hline
\end{tabular}

Table 3. Lightning strike parameters.

\begin{tabular}{ccc}
\hline Parameters & First bow & Return bow \\
\hline$I_{01}(\mathrm{kA})$ & 28 & 10.7 \\
$\tau_{11}$ & 1.8 & 0.25 \\
$\tau_{21}$ & 95 & 2.5 \\
$n_{1}$ & 2 & 2 \\
$I_{02}$ & - & 6.5 \\
$\tau_{12}$ & - & 2 \\
$\tau_{22}$ & - & 230 \\
$n_{2}$ & - & 2 \\
\hline
\end{tabular}


- On the other hand, theory shows and experience confirms that the shape of the lightning discharge current has a time evolution of the form $i(t)=I_{o}\left(\mathrm{e}^{-A t}-\mathrm{e}^{-B t}\right)$. For an atmospheric discharge characterized by the following: $I_{o}=10 \mathrm{kA}, A=1.4 \times 10^{4} \mathrm{~S}^{-1}$ et $B=6 \times 10^{5} \mathrm{~S}^{-1}$. Figure 10 below, obtained by simulation under Matlab environment, and shows the current pattern at the base of lightning channel.

- The space-time distribution of lightning current obtained by simulation under Matlab environment (see Figure 11) considers the case where the lightning current has the characteristics such as: $I_{o}=10 \mathrm{kA}, A=1.4 \times 10^{4} \mathrm{~S}^{-1}$, $B=6 \times 10^{5} \mathrm{~S}^{-1}, Z$ varying from 0 to $40 \mathrm{~m}, t$ varies from 0 to $30 \mu \mathrm{s}$ and $v=3 \times$ $10^{6} \mathrm{~m} / \mathrm{s}$.

- In the case of a lightning with the characteristics such as $I_{o}=25 \mathrm{kA}$, $A=1.4 \times 10^{4} \mathrm{~S}^{-1}$ et $B=6 \times 10^{5} \mathrm{~S}^{-1}$ and a wave impedance line: $Z_{c}=50 \mathrm{Ohm}$, Figure 12 below shows the time evolution of the lightning induced voltage on the phase conductor, obtained by simulation under Matlab environment.

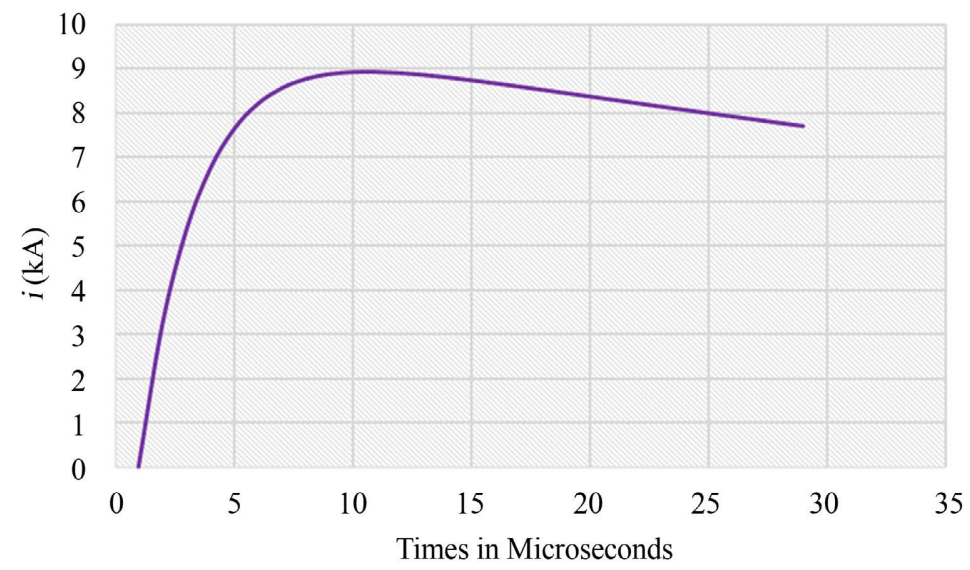

Figure 11. Calculation result: Surge of lightening current.

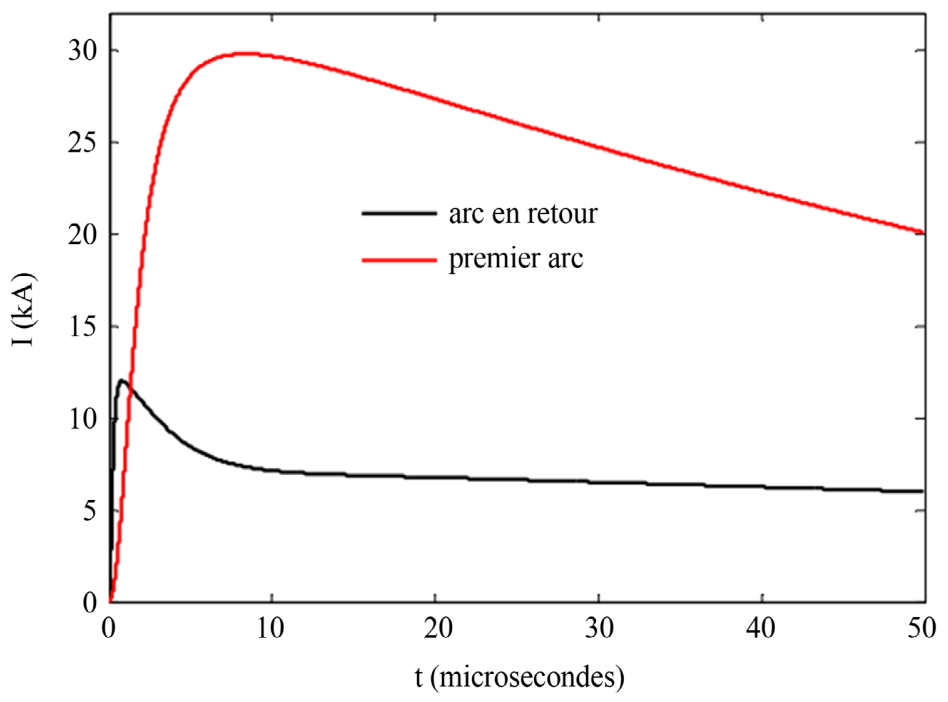

Figure 12. Results published by J.P NZURU NSEKERE in 2009 [36]. 


\subsection{Results}

See Figures 13-16.

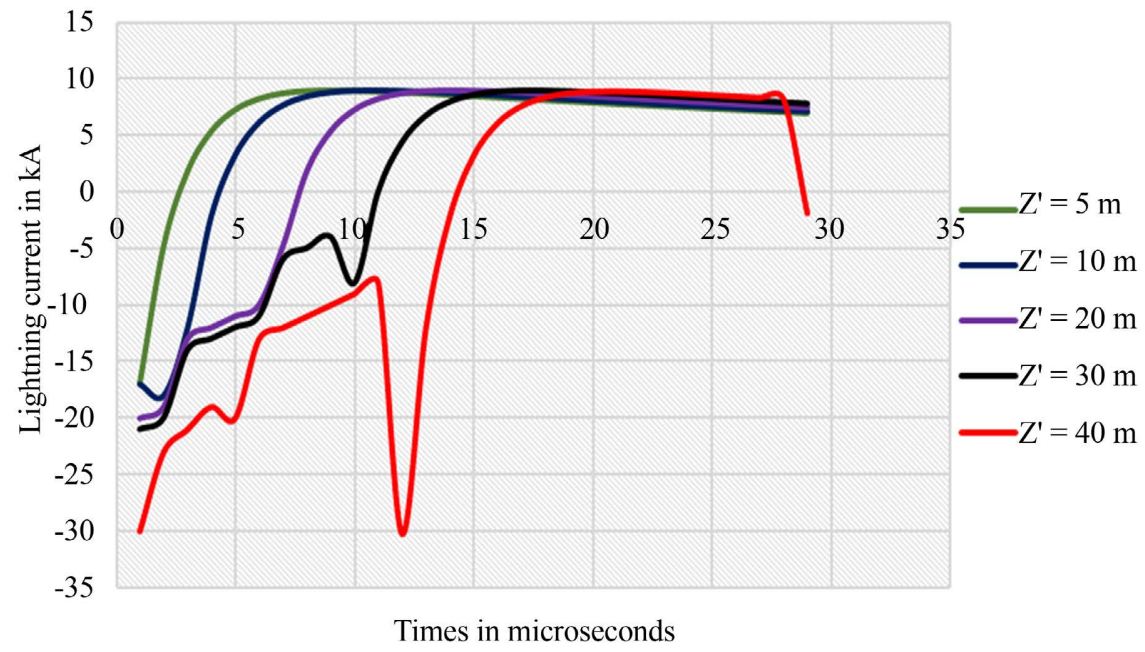

Figure 13. Spatio-time distribution of lightning current.

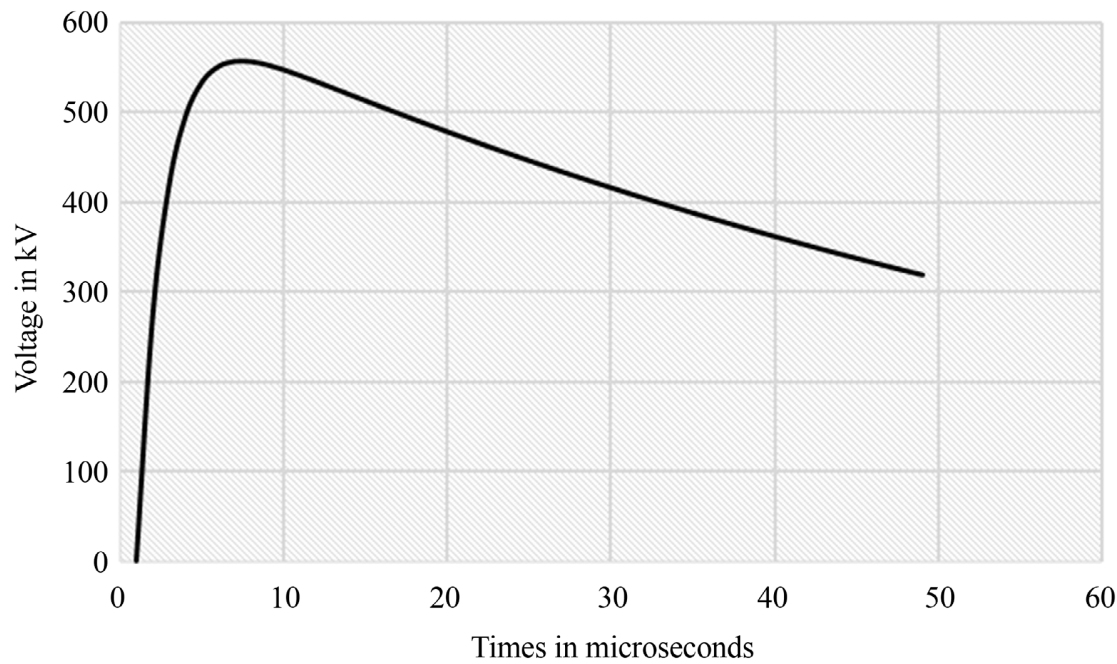

Figure 14. Calculation result: temporal evolution of the atmospheric over voltage included in the phase conductor.
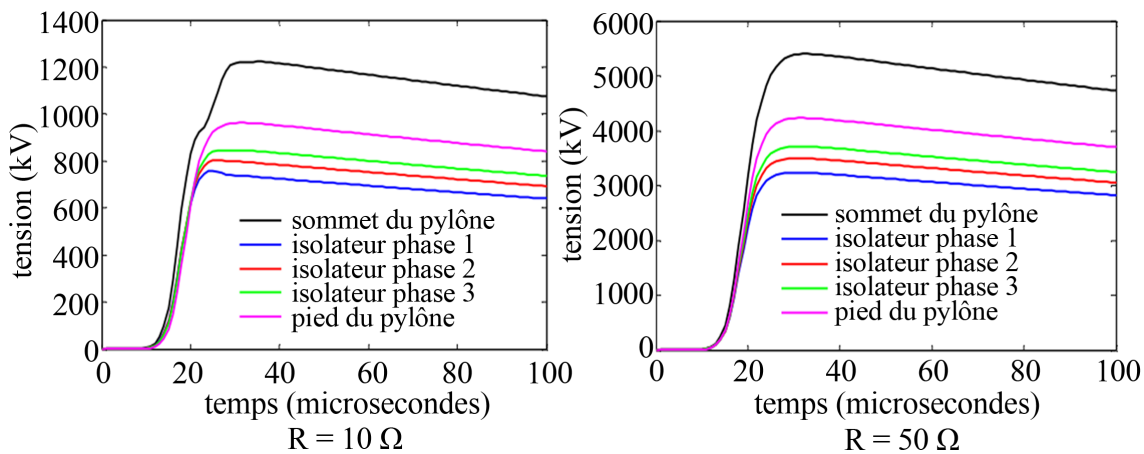

Figure 15. Results published by J. P NZURU NSEKERE in 2009 [36]. 
The simulation of the influence of atmospheric overvoltage on the transportable power of an HV power line implements Equations (35) and (36). In this case, the transportable power depends only on the line reactance. In the case where the lightning current is characterized by the following parameters $I_{o}=10 \mathrm{kA}$, $A=1.4 \times 10^{4} \mathrm{~S}^{-1}$ and $B=6 \times 10^{5} \mathrm{~S}^{-1}$ and the allowed overvoltage being $23 \%$ of the nominal voltage, the high voltage line of $200 \mathrm{~km}$ length and having the characteristics such as Length: $L=200 \mathrm{~km}$; Linear Reactance $x_{0}=0.2142 \mathrm{Ohms} / \mathrm{km}$; Frequency: $f=50 \mathrm{~Hz}$ and nominal voltage of the line $u_{s}=220 \mathrm{kA}$. Figure $16 \mathrm{be}-$ low gives the result of the numerical simulation under Matlab of the line behavior during discharge.

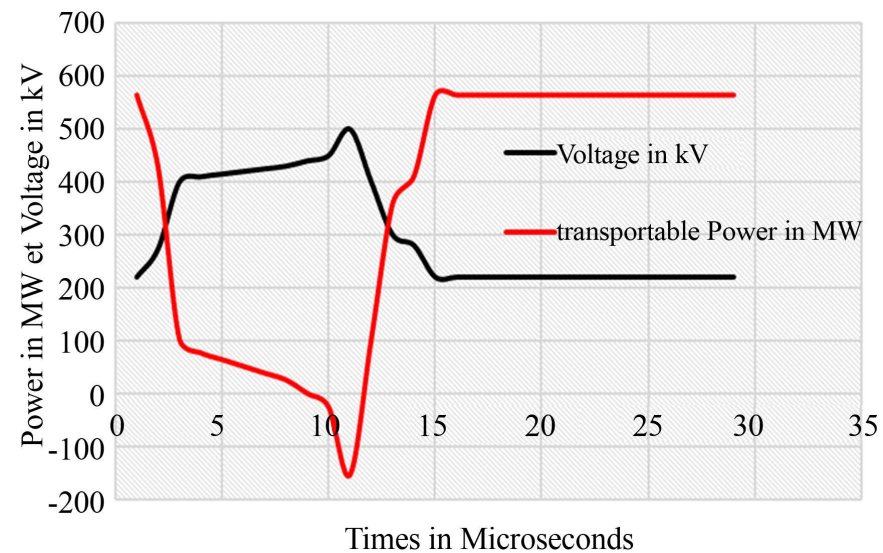

Figure 16. Behavior of the power of a line during atmospheric overvoltage.

\section{Discussion of the Results}

Transmission lines are the most exposed to disturbances during a lightning discharge. The study of their transient behavior is generally based on the theory of transmission lines with electrical components such as $R, L, C$ and $G$. Several equations exist for their modelling depending on the shape and characteristics of the material used in the installation. In order to obtain the results of different simulations, the bi-exponential and engineering models have been chosen and used for the analytical modeling of the problem that is the subject of this paper. For this purpose, the following results were obtained:

Figure 13 shows that the transmissible power of a transmission line is a parabolic function of the source voltage. With the experimental data taken from the work of Dennis and Pierce, Figure 10 shows that, the current flow at the base of the lightning channel is of an impulse nature, and is characterized by the rise and fall time, the result obtained is close to the experiments proposed by Nucci to reproduce a typical lightning wave obtained by measurements [Nucci, C. A., Diendorfer G., Uman M., Rachidi F., Ianoz M., and Mazzetti C., 1990] [25] see Figure 14. Figure 11 represents rather the temporal evolution of the voltage induced by lightning in the phase conductor and also shows that, this voltage is directly proportional to the temporal variation of the disturbance current, this result approaches those presented in the work proposed by J. P NZURU NSEKERE 
in 2009 [36] see Figure 14.

Figure 15 on the other hand describes the spatio-temporal distribution of the lightning current and shows that at heights below the front of the return arc i.e. $z^{\prime} \leq v . t$, this current is equal to the current at the base of the lightning channel, and at heights above the front of the return arc in the case where $z>v$. $t$, this current is negative or even zero. And finally, Figure 16 shows that during the period from 0 to 2 microseconds the power transportable by the line is maximum i.e. $565 \mathrm{MW}$ when the load voltage is equal to the source voltage i.e. $u_{s}=u_{c}$ $=220 \mathrm{kV}$. But in case of overvoltage, during the period from 2 to 15 microseconds, a transient regime appears the power that can be carried by the line, this is only transient but can be a problem in the future, cause an increase in the frequency of unscheduled outages in the case of lines operating without the power reserve margin. These outages are responsible for the loss of undistributed kilowatt-hours, i.e. loss of income for the electric power transmission and distribution company. For this purpose, in case of strong overvoltage, the HV line must be equipped with protection devices that are more efficient than if the line is resistive or inductive without compensation.

\section{Conclusion}

At the end of our work we analyzed the behavior of a HV line during an atmospheric discharge and evaluated the spatio-temporal distribution of the lightning current wave. The emphasis was put on a compensated HV inductive line, especially since most of the lines actually operating are compensated. We were interested in the study of the space-time distribution of the lightning current wave in order to model the radiated electromagnetic field and to examine the influence of the overvoltage induced by the atmospheric discharge on the transportable power of a High Voltage AC transmission line. After theoretical considerations where we established some mathematical models that helped us to define the problem, we took a practical case to make the simulation, the line being fed by a source of $220 \mathrm{kV}$, it results that, the lightning current being of impulse nature is characterized by the time of rise and fall. The power drop only occurs in transient regimes or when the overvoltage reaches $23 \%$ of the nominal voltage.

\section{Conflicts of Interest}

The author declares no conflicts of interest regarding the publication of this paper.

\section{References}

[1] Aguet, M. and Morf, J.J. (1981) Electricity, Treaty of Electricity, Volume XII. Polytechnic Press of Lausanne, Lausanne.

[2] Aguet, M. and Ianovici, M. (1982) Haute Tension, Treaty of Electricity, Volume XXII. Presse Polytechnique de Lausanne, Lausanne.

[3] Gardiol, F. (1996) L’Electromagnétisme, Treaty of Electricity, Volume III. Lausanne Polytechnic Press, Lausanne. 
[4] Haddad, A. and Warne, D. (1996) Advances in High Voltage Engineering. The Institution of Engineering and Technology, London.

[5] Begamudre, R.D. (1986) Extra High Voltage AC Transmission Engineering. New Age International Publishers, New Delhi.

[6] Denno, K. (1922) High Voltage AC Transmission Engineering. CRC Press, Boca Raton.

[7] Roy, G., Gary, C. and Hutzler, B. (1988) The Dielectric Properties of Air and Very High Voltages. Eyrolles, Paris.

[8] Gary, C. (1995) Crown Effect in Alternative Tension.

[9] Pelissier, R. (1975) Les reseaux d'energie Electrique, Volume 4, Propagation of Electric Waves on Power Lines, Dunod Technique.

[10] Ianovici, M. and Morf, J.J. (1983) Electromagnetic Compatibility. Presses Polytechniques Romandes, Lausanne.

[11] Aguet, M. and Morf, J.J. (1987) Electrical Energy. EPFL Electricity, Electronics and Electrical Engineering Treatise. Presses Polytechniques Romandes, Lausanne, 335 p.

[12] Guerrieri, S., et al. (1998) On the Influence of Elevated Strike Objects on Directly Measured and Indirectly Estimated Lightning Currents. IEEE Transactions on Power Delivery, 13, 1543-1555. https://doi.org/10.1109/61.714865

[13] Yang, G., et al. (2017) Evaluation of Lightning Current from Magnetic Field Based on Deconvolution Method. IEEE Transactions on Electromagnetic Compatibility, 60, 679-684. https://doi.org/10.1109/TEMC.2017.2747514

[14] Uman, M.A. and McLain, D.K. (1970) Lightning Return Stroke Current from Magnetic and Radiation Field Measurements. Journal of Geophysical Research, 75, 5143 5147. https://doi.org/10.1029/JC075i027p05143

[15] Affolter, J.F. (2000) Energies et Réseaux Electriques IESE, EIVD Handout of Advanced Courses.

[16] Allab, K. (1984) Elements of Mathematical Analysis-Function of a Real Variable. Scientific Schools.

[17] Spiegel, M.R. (1975) Schaum's Outline of Theories and Problems of Advanced Calculus. McGraw-Hill, New York, 160-165.

[18] Rachidi, F. (2002) Electromagnetic Effects of Lightning on Electric Lines, Electromagnetic Compatibility EMC, Course Handout, LRE, EPFL.

[19] de Coulon, F. and Jufer, M. (1984) Introduction to Electrical Engineering, Treatise on Electricity, Electronics and Electrical Engineering from EPFL. Presses Polytechniques Romandes, Lausanne, $347 \mathrm{p}$.

[20] Lasne, L. (2004) Electrical Engineering: Basics and Electrical Network. University of Bordeaux, Department of Electronics-Electrical Engineering-Automation, Bordeaux.

[21] Van Cutsem, T. (1998) Electrical Power Systems. University of Liège, Department of Electricity, Electronics and Computer Science (Institut Montefiore), Liège.

[22] Cooray, V. (2003) The Lightning Flash. IEE Press, London. https://doi.org/10.1049/PBPO034E

[23] Guemri, B. (2004) Study and Analysis of the Electromagnetic Field-Transmission Line Coupling. Magister's Thesis, USTO-MB, Oran, Algeria.

[24] Bermudez Arboleda, J.L. (2003) Lightning Currents and Electromagnetic Fields Associated with Return Strokes to Elevated Strike Objects. These Doctorate es Science, EPFL, Lausanne.

[25] Nucci, C.A., Diendorfer, G., Uman, M., Rachidi, F., Ianoz, M. and Mazzetti, C. (1990) Lightning Return Stroke Current Models with Specified Channel-Base Current: 
A Review and Comparison. Journal of Geophysical Research: Atmospheres, 95, 20395-20408. https://doi.org/10.1029/JD095iD12p20395

[26] Ianovici, M. and Morf, J.J. (1985) Electromagnetic Compatibility. 2nd Edition, Presses Polytechniques Romandes, Lausanne.

[27] Cocquerelle, J.L. (1999) C.E.M. and Power Electronics. Editions Technip, Paris.

[28] Degauque, P. and Hamelin, J. (1990) Electromagnetic Compatibility: Radioelectric Noise and Disturbance. Technical and Scientific Collection of Telecommunications. Éditions Dunod, Paris.

[29] Orzan, D. (1998) External and Internal Coupling between an Electromagnetic Field and a Network of Multi-Wire Lines. Doctoral Thesis, Ecole Polytechnique Fédérale de Lausanne (EPFL), Lausanne.

[30] Sommerfeld, A. (1909) Über die Ausbreitung des wellen in der drahtlosen Telegraphie. Annalen der Physik, 333, 665-736.

https://doi.org/10.1002/andp.19093330402

[31] Rachidi, F. (1991) Electromagnetic Effects of Lightning on Aerial Transmission Lines, Modeling and Simulation. Doctoral Thesis, Swiss Federal Institute of Technology in Lausanne, Lausanne.

[32] Dib, D. (1997) Study and Analysis of the Propagation of Atmospheric Overvoltages in HV Lines. Thesis of Magister, University of Annaba, Annaba.

[33] Gary, C., Dragan, G. and Critescu, D. (1978) Attenuation of Propagation of Surges under the Influence of the Corona Effect, Extract from CIGRE, Group 33-13.

[34] Kiessling, F., Nefzger, P., Nolasco, J.F. and Kaintzyk, U. (2003) Overhead Power Lines: Planning, Design, Construction. Springer-Verlag, Berlin.

https://doi.org/10.1007/978-3-642-97879-1

[35] Gary, C. (2014) The Dielectric Properties of Air and Very High Voltages. Editions Eyrolles, Paris.

[36] Nsekere, J.P.N. (2009) Contribution to the Analysis and Realization of the Earthing of Electrical Installations in Tropical Regions. Doctoral Thesis, University of Liège, Faculty of Applied Sciences, Liège. 